Original Research Paper

\title{
Present a Mechatronic System Having Able to Determine the Concentration of Carotenoids
}

\author{
${ }^{1}$ Raffaella Aversa, ${ }^{2}$ Eleni Buzea, ${ }^{2}$ Relly Victoria V. Petrescu, \\ ${ }^{1}$ Antonio Apicella, ${ }^{2}$ Marin Neacșa and ${ }^{2}$ Florian Ion T. Petrescu \\ ${ }^{I}$ Advanced Material Lab, Department of Architecture and Industrial Design, \\ Second University of Naples, 81031 Aversa (CE) Italy \\ ${ }^{2}$ IFToMM, ARoTMM, Bucharest Polytechnic University, Bucharest, (CE) Romania
}

Article history

Received: 27-11-2016

Revised: 03-12-2016

Accepted: 06-12-2016

Corresponding Author: Florian Ion T. Petrescu IFToMM, ARoTMM, Bucharest Polytechnic University, Bucharest, (CE) Romania

Email: petrescuflorian@yahoo.com

\section{Introduction}

Civilization and progress have brought us many benefits. Today we not live in the field, in forests or in the caves. We have houses specially designed with air conditioning in summer and winter heating. We have transport quickly by any direction and wireless communications. We can't yet devise spaceships high speed and autonomy and satellites to locate us with great precision habitable planets. I mean that today, we (considered to be a civilization top) we can only ask for a Galactic expansion of humanity. We don't have real possibilities to accomplish although current technologies would allow it. When we have opened "Pandora's box"

\begin{abstract}
Civilization besides its major advantages (undisputed) brought us some unhealthy habits which unfortunately have managed to penetrate deeply within several generations on the current planetary populations producing great damages to its health. Perhaps it would be the right time to and more people learning to live healthy, to feed ourselves healthy, let's try aging. The role to annihilate them is fulfilled by the antioxidants. Antioxidants are a group of compounds produced by the organism or that body to maintain health and vigor, especially during the last decades of life. Antioxidants network offers the advantage of avoiding premature death caused by these deficiencies and sometimes even cure those diseases. The methods generally used for the determination of carotenoids extracted from a natural environment are based on absorption spectrophotometry. These methods may be relatively precise but require a relatively long preparation of the sample to be analyzed. Indeed, because of the other pigments often present in the samples analyzed and extracted at the same time as the carotenoids, the analysis is not very precise on the crude acetone extract, chlorophylls in particular are often troublesome. Saponification and
\end{abstract} Keywords: Biomaterials, Bioengineering, Biotechnologies, Biophotonic Scanner, Carotenoids, Antioxidants, Free Radicals, Slowing Aging, Biochemical Processes we have overflowed the humanity by the good and by the all evil, leaving them to tumbling out. Unfortunately we haven't today political capacity to solve all essential issues of humanity. However many of them have begun to be taken into account. How do we want to have more money for the research concerned eradicate major diseases and for the extension of life, when now we have multiplied too much and the planet on which we are now all closed, can only lead us all. The methods generally used for the determination of carotenoids extracted from a natural environment are based on absorption spectrophotometry. These methods may be relatively precise but require a relatively long preparation of the sample to be analyzed. Indeed, because of the other 
pigments often present in the samples analyzed and extracted at the same time as the carotenoids, the analysis is not very precise on the crude acetone extract, chlorophylls in particular are often troublesome. Saponification and different extraction methods are then necessary to purify the solution, which inevitably leads to loss of carotenoids, thus an error in the assay. Raman resonance spectrometry has been used successfully for the detection and identification of algae or bacteria in water and for determining the concentration of carotenoids in marine phytoplankton. One can generalize this method to other species, in particular benthic macrofauna species and to compare the results with those obtained from absorption spectrometry.

By exciting the Raman spectrum of a carotenoid within its visible absorption band $\left(\pi->\pi^{x}\right.$ transition of the polyenic chain), a very large increase in line intensity attributable to the polyenic chain is observed; It can be $10^{6}$. The Raman spectrum thus obtained is characteristic of the only carotenoids present in the solution, because under these conditions the intensity of the Raman lines characteristic of the non-absorbent species or of the other pigments are much too low to be detected. The direct and specific analysis of the carotenoids within a system as complex as a solution obtained after extraction, is then possible. The intensity of the resonance Raman lines is proportional to the concentration of the pigment at a given excitation wavelength. A reference substance in solution with the carotenoids (or the solvent itself) serves as an internal standard. The ratio of the intensity of a carotenoid line to the intensity of a line of the solvent, leads through a calibration prior to the carotenoid concentration in the solution. Visible adsorption is not sufficiently selective and pigments other than carotenoids can alter the measured optical density. Each carotenoid has its own excitation profile (variation of coefficient of exaltation as a function of the excitation wavelength) and, in the case of a given concentration mixture the measured intensity varies according to of the relative proportion of constituents. The excitation profile of each carotenoid, which is the main source of error of this method, can be used to determine the majority compound in a mixture and thus considerably reduce the error caused by a bad reference choice.

A major medical problem is that of free radicals present in the human body. They are formed in the human cells and have the role to destroy certain pathogenic bacteria. Their presence is permanent and required in the human body. But when they multiply too much might get out of control and can be very toxic to human body. So as long as they are check of the cells and the immune system radicals fulfill only the role good and are friendly. Serious problems produce them only when they are free to cell or body. It has been shown that and free radicals in the body can be put under control by antioxidants present themselves in the human body.
Free Radicals adversely affecting the DNA cells; are adversely affected mainly heart cells, brain cells and some immune system; it can be altered the genetic material of a cell; as a result changes occur in the structure of a protein and after the immune system considers the cell a foreign substance and are trying to destroy it. Free Radicals can damage and protective membrane of cells, causing their death and in this way. Any cell receives approximately ten thousands of attacks on day from the free radicals. A free radical is an atom or group of atoms containing a lone electron noncompensated; it can live only one fraction of a second.

The oxygen is necessary human metabolism and to convert nutrients into energy; the most known free radicals are oxygen derivatives, such as superoxide, hydroxyl, hydrogen peroxide, nitric oxide, etc. Free radicals are formed by a number of different metabolic processes such as breaking down the fat molecules stored for their use as an energy source, by exposure to sunlight or X-ray, or in the presence of harmful chemicals (from cigarette smoke, from the exhaust gases, from polluted atmosphere).

But immune system produces and the radical free useful, to destroy viruses and bacteria. When the production of free radicals in the body is too high there is a defect of cells and a multiplication of free radicals above normal level (that can be controlled by the body). If free radicals activity is more intense than the work of neutralization of the antioxidants, result in an imbalance called "oxidative stress", which can cause major chronic degenerative diseases, such as: Cardiovascular disease, cancer, mental disease, body aging. Today a large majority of the population of advanced countries from the point of view industrialtechnological suffers because of diseases caused by deficiencies of the network of antioxidants. The network of antioxidants offers the advantage to avoid premature death caused by these deficiencies and sometimes even cure of the disease concerned. Network components of antioxidants are vitamin $\mathrm{C}$, vitamin $\mathrm{E}$, as well as nutrients needed to produce body following antioxidants: Lipoic acid, coenzyme Q10 (Co Q10) and glutathione (Packer and Colman, 1999; Packer, 2002). It is considered that the most important antioxidants that the body can produce usually are: Superoxide Dismutase (SOD), glutathione peroxidase, catalase and methionine reductase.

\section{Materials and Methods}

\section{The Relation between Network Status of Antioxidants Cells of human Body and the Concentration of Carotenoids from Skin}

Carotenoid molecules degrade in the process of neutralizing free radicals. 
A recent study led by Arne Svilaas concluded that these carotenoids are an accurate indicator of the level of antioxidants. Svilaas evaluated the content of antioxidants in the diet of more than 2670 adults and measured the serum levels of antioxidants more than 61 patients for 7 consecutive days; found that is greater the ability of carotenoids to predict the other antioxidants level than predictive ability of alpha, beta and gammatocopherols (vitamin E), as well as that of glutathione; this result is explained by the fact that network of antioxidants protects (more efficient) the body cells from free radical attacks and therefore carotenoids molecules degrade less in the process of neutralizing free radicals; such molecules content of carotenoids in the skin increases, because they are neutralized to a lesser extent by free radicals; Raman method is effective that direct measurement of carotenoids in the skin, without the inconvenience of blood or urine samples.

Clinical studies have revealed that there is a direct correlation between antioxidant levels and general health and immunity of the human body with a relevance factor of $84 \%$.

\section{Measurement of Carotenoids Concentration from the Skin}

Biophotonic scanner applies a noninvasive method of measuring the level of antioxidants; measures the level of carotenoid antioxidants, safely, using Raman spectroscopy. Biophotonic scanner emits a laser light with a wavelength of $473 \mathrm{~nm}$; if the laser light meets a carotenoid molecule, it generates a green light with a wavelength of $510 \mathrm{~nm}$; this is captured by an ultra light detector connected to a computer, which analyzes the amount of green light indicates the concentration of carotenoids in the skin, called Skin Carotenoid Score (SCS). 10,000 units correspond to a carotenoid concentration of $0.04 \mathrm{mmol} \mathrm{mL}^{-1}$ of blood.

A carotenoid score of less than 20000 units reflects a very small pool of health, high risk of disease due to a wrong diet; carotenoid score greater than 20000 and less than 30000 units reflect a reserve of low health; carotenoid score greater than 30000 and less than 40000 units reflects a satisfactory health reserve, between 40000 and 50000 better and more than 50000 health reserve is considered very good and the risk of diseases listed above is very small (Bojovic and Stojanovic, 2005).

Dr. Oz has "75,000 units, because he eat calculated as anyone could do it, not eat anything special, but has a different lifestyle in the sense of eating another kind of food."

High values in the level of carotenoids dramatic increase chances for the fight with a cancer which may threaten life to everyone at a certain point in time. For example low carotenoids level may be caused by an increased consumption of pastry products.
The use of Raman spectroscopy for the determination of carotenoids in the skin has been validated by independent studies carried out and the teams led by Bernstein (Gellermann et al., 2002; Bernstein et al., 2002; Bernstein, 2002), or Ermakov et al. (2001), or Hata et al. (2000).

The new S3 scanner will dramatically improve the user experience, being smaller, faster, battery-powered and integrated with IOS wireless capability.

By measuring the stable level of carotenoid antioxidants in your skin and generating your Skin Carotenoid Score, the Scanner provides a more accurate and reliable biomarker of your overall antioxidant health status than other methods of measuring antioxidants. Getting your Skin Carotenoid Score makes you aware of the antioxidant levels in your body-and gives you the push you need to improve your overall antioxidant health.

\section{Quality Control of Fruit and Vegetables with Portable Raman Spectrometers}

Portable Raman systems achieved rapid identification of substances and determine their concentration in the analyzed material. Raman technique advantages are: Measurement technology is non-destructive; give very specific information at the molecular level; wavelength for the excitation of molecules can be chosen optimal depending on the nutrient, antioxidant, which is analyzed; optical fibers can be used for illumination or detection; samples analyzed may have small size, on the order of millimeters; radiation can pass through thin glass or plastic transparent or translucent, if this requirement is imposed on the method for determining the concentration of the nutrient in question; samples preparation is easy to perform and can be automated; Raman measurement systems are safe, easy to use, compact, robust and portable; quantitative analysis with statistical evaluation "Teach/Match" can use the spectra libraries specific to each area of use. For example, Xantus-Mini Raman spectrometer with 1064 nm wavelength has an I-phone interface.

\section{Determination of Carotenoids from Fruits and Vegetables}

First, the action mechatronics profitable to determine the content of antioxidants in fruits and vegetables, we address the determination of ascorbic acid in fruit and vegetables. Several methods are known for the determination of ascorbic acid, such as: A-Methods based on reducing properties of vitamin $\mathrm{C}$, such as iodometric method. b-For the evaluation of total antioxidant capacity of commercial fruit juices by the spectrophotometric method was used redox couple DPPH (2,2-diphenyl-1-picrylhydrazyl)/DPPH (2,2diphenyl picryl hydrazine 1); Followed DPPH 
absorbance decrease (Pisoschi et al., 2009). cDetermination of ascorbic acid by voltammetry carried out with carbon electrodes (Pisoschi et al., 2011). d-One method used to determine fast and simple ascorbic acid in pharmaceutical products, fruit juices, soft drinks and serum is chemiluminescent reaction in alkaline solution of lucigenin with photooxidation products of ascorbic acid toluidine blue sensitized (Pérez-Ruíz et al., 1995).

These methods require costly preparation of samples that can be and a source of errors. Therefore it would be useful to investigate feasibility of determining the concentration of vitamin $\mathrm{C}$ in foods with Raman spectrometers (Wellburn, 1994).

\section{Food Safety Risk Minimization}

Management System in food safety SQF (SMSA) is designed to give organizations a rigorous food safety risk management system; as a result, the market offering safe products and offers customers a recognized food safety certification. SQF Code contains requirements for integrated processes that work together to control and minimize food safety risks.

Mission of nutrient control laboratories is to provide leadership and promote international cooperation in the development of databases of nutrients; State authority requires methods to acquire, assess, implement and disseminate data on the composition of foods available in the market.

Control devices should be purchased and used by the state authority of each country.

\section{Results and Discussion}

\section{Support Systems for Group Decision on Minimize Food Safety Risk}

To minimize the risk of malnutrition, it is useful to adopt rational decisions with group decision support systems; these systems can be integrated into CLOUD COMPUTING, for that segment of the market to be as high as possible and statistical evaluations to be more precise.

An essential criterion of decision recommended is "internal rate of return" which must be large enough to allow the remuneration of capital is greater than if the capital were placed in a bank (see the relationship 1):

$$
V_{o}=\sum_{i=1}^{n} \frac{F i}{(1+r)^{i}}+\frac{V_{r}}{(1+r)^{n}}
$$

Where:

$$
\begin{aligned}
n= & \text { Life of the instruments used to determine the } \\
& \text { antioxidant network, in order to reduce the } \\
& \text { risk of malnutrition } \\
(1+r)= & \text { Internal rate of return of financial resources }
\end{aligned}
$$

$$
\begin{aligned}
V_{o}= & \text { Initial cost of the investment } \\
V_{r}= & \text { Residual value of the instruments for the } \\
& \text { determination of antioxidant network } \\
F i= & \text { Profit and depreciation for the year of } \\
& \text { operation of instruments for determining the } \\
& \text { antioxidant network }
\end{aligned}
$$

For that the profit is as high as possible is required intensive use of instruments. For this must that initially the price to be low enough to attract users. One can call on companies already performing Raman spectrometry control services.

\section{Carotenoids}

Carotene Def. DEX Pigment red, orange found in foods of plant and animal foods. Carotenoids are pigments that give color of egg yolk, tomatoes, all fruit and flowers. They are found in shellfish, some species of fish, bird feathers and insects. Carotenoizii play a role of antioxidants acting against free radicals against premature aging and counter of the spots on the skin. Carotenoids facilitate communication of cells and can be converted into vitamin A when necessary (especially beta-carotene). The daily requirement is $2-4 \mathrm{mg}$.

Carotenoids needed of human body are: Betacarotene, lutein, zeaxanthin, lycopene. Beta-carotene is found in dark yellow-orange fruits and vegetables such as carrots, apricot (Marx et al., 2000).

Beta-carotene is one of over 600 carotenoids that are found in nature. Beta-carotene is converted to vitamin A and is the most important source of provitamin A from food. Acts as an antioxidant with vitamins $\mathrm{E}$ and $\mathrm{C}$ and can reduce the risk of chronic diseases.

Beta-carotene also plays important role in protecting your skin from the harmful effects of ultraviolet radiation.

The most important sources of carotenoids are carrots, spinach, broccoli, pumpkin, lettuce, sweet potatoes, red peppers, rosehip, sour, bitter cherries, apricots. Lutein is found in fruits and green vegetables, red and yellow, such as peas, grapes, cabbage, pepper, oranges, kiwi.

Lutein is one of the two carotenoids located in the macula of the eye (part of the retina which perceives light and provides maximum visual acuity and is able to filter out harmful blue light and thereby reduce the risk of cataracts and maculopapular dystrophy two of the causes of blindness).

All these carotenoids participate in the formation of vitamin $\mathrm{A}$ in the body which in turn forms Rhodopsin. In the absence of rhodopsin appear visual disturbances and in advanced forms occur conjunctiva and corneal lesions. Carotenoids are obtained mainly from fruits, vegetables and fresh herbs (Fig. 1). 


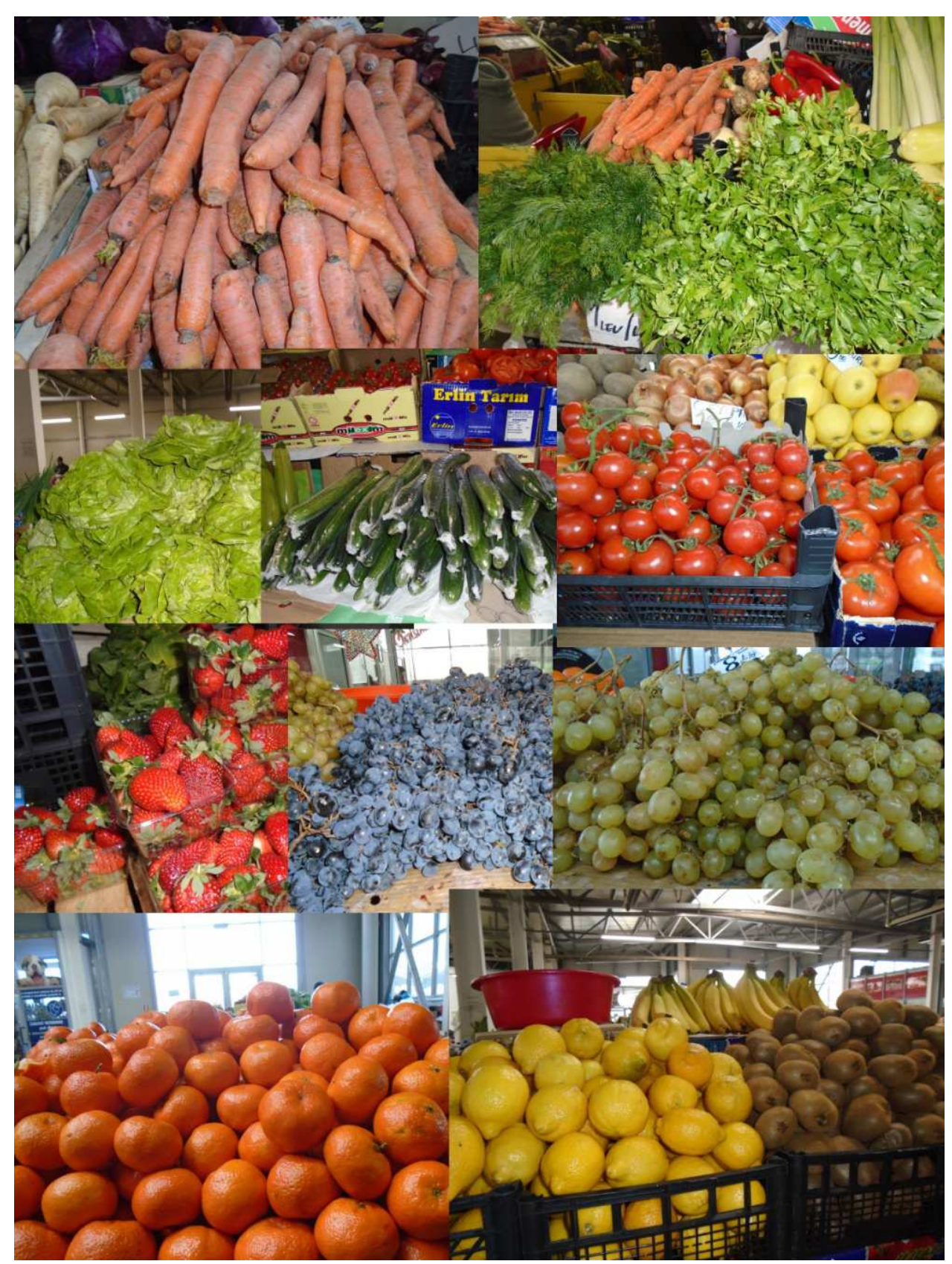

Fig. 1. Natural sources of carotenoids

\section{Conclusion}

Determination of antioxidants network in food with Raman spectroscopy can be more effective than pursuing total antioxidant capacity of foods outside the bodies. Determination of carotenoids from fruits and vegetables with Raman spectrometers it would be a very useful method in the future. The development of modern methods and fast like this still takes place with or without the consent of many existing opposing forces on the planet today who do not want the development of mankind and in this way simply because they follow other interest groups (usually financial). Any important contribution to people's health and extend life with health is a serious scientific contribution and deserve to be taken into account. The best way to prolong life is to strengthen the antioxidant defense network that can control the forces that drive the aging process. The main antioxidants that must be consumed daily are vitamins $\mathrm{C}, \mathrm{E}$ and $\mathrm{A}$. This is done easiest by a diet rich in fruits, vegetables and fresh greens. 


\section{Acknowledgement}

This text was acknowledged and appreciated by Assoc. Pro. Taher M. Abu-Lebdeh, North Carolina A and $\mathrm{T}$ State Univesity, United States, Muftah H. ElNaas PhD MCIC FICCE QAFCO Chair Professor in Chemical Process Engineering Gas Processing Center College of Engineering Qatar University, whom we thanks and in this way.

\section{Author's Contributions}

All the authors contributed equally to prepare, develop and carry out this manuscript.

\section{Ethics}

This article is original and contains unpublished material. The corresponding author confirms that all of the other authors have read and approved the manuscript and no ethical issues involved.

\section{References}

Bernstein, G., 2002. Measurement of carotenoids in the living primate eye using resonance Raman spectroscopy. Meth. Mol. Biol., 196: 321-9. DOI: $10.1385 / 1-59259-274-0: 321$

Bernstein, P.S., D.Y. Zhao, S.W. Wintch, I.V. Ermakov and R.W. McClane et al., 2002. Resonance Raman measurement of macular carotenoids in normal subjects and in age-related macular degeneration patients. Ophthalmology, 109: 1780-7. PMID: 12359594

Bojovic, B. and J. Stojanovic, 2005. Chlorophyll and carotenoid content in wheat cultivars as a function of mineral nutrition. Arch. Biol. Sci., 57: 283-290. DOI: $10.2298 / \mathrm{ABS} 0504283 \mathrm{~B}$

Ermakov, I.V., M. Sharifzadeh, M. Ermakova and W. Gellermann, 2001. Resonance Raman detection of carotenoid antioxidants in living human tissue. Opt. Lett., 26: 1179-1181. DOI: 10.1117/1.2139974

Gellermann, B., I.V. Ermakov, M.R. Ermakova, R.W. McClane and D.Y. Zhao et al., 2002. In vivo resonant Raman measurement of macular carotenoid pigments in the young and the aging human retina. J. Opt. Soc. Am. A, 19: 1172-86.

DOI: $10.1364 /$ JOSAA.19.001172
Hata, T.R., T.A. Scholz, I.V. Ermakov, R.W. McClane and F. Khachik et al., 2000. Non-invasive Raman spectroscopic detection of carotenoids in human skin. J. Invest. Dermatol., 115: 441-8. DOI: 10.1046/j.1523-1747.2000.00060.x

Marx, M., A. Schieber and R. Carle, 2000. Quantitative determination of carotene stereoisomers in carrot juices and vitamin supplemented (ATBC) drinks. Food Chem., 70: 403-408. DOI: $10.1016 / \mathrm{S} 0308-8146(00) 00096-0$

Packer, L. and C. Colman, 1999. The Antioxidant Miracle: Put Lipoic Acid, Pycnogenol and Vitamins $\mathrm{E}$ and $\mathrm{C}$ to Work for You. 1st Edn., John Wiley Sons, Inc., ISBN-10: 0471353116, pp: 256.

Packer, L., 2002. The antioxidant Vitamins C and E. The American Oil Chemists Society.

Pérez-Ruíz, T., C. Martínez-Lozano and A. Sanz, 1995. Flow-injection chemiluminometric determination of ascorbic acid based on its sensitized photooxidation. Anal. Chim. Acta, 308: 299-307. DOI: 10.1016/0003-2670(94)00527-S

Pisoschi, A.M., M.C. Cheregi and A.F. Danet, 2009. Total antioxidant capacity of some commercial fruit juices: electrochemical and spectrophotometrical approaches. Molecules, 14: 480-493. DOI: $10.3390 /$ molecules 14010480

Pisoschi, A.M., A. Pop, G.P. Negulescu and A. Pisoschi, 2011. Determination of ascorbic acid content of some fruit juices and wine by voltammetry performed at $\mathrm{Pt}$ and carbon paste electrodes. Molecules, 16: 1349-1365.

DOI: 10.3390/molecules16021349

Wellburn, A.R., 1994. The spectral determination of chlorophylls a and b, as well as total carotenoids, using various solvents with spectrophotometers of different resolution. J. Plant Physiol., 144: 307-313. DOI: 10.1016/S0176-1617(11)81192-2 